\title{
Flow of a viscoelastic shear-thinning fluid between two concentric rotating spheres
}

\author{
Eric Lee, Yun-Hai Lee, Yao-Tzung Pai, Jyh-Ping Hsu * \\ Department of Chemical Engineering, National Taiwan University, Taipei, Taiwan 10617, ROC
}

Received 11 May 2001; received in revised form 14 September 2001; accepted 18 September 2001

\begin{abstract}
The flow of a viscoelastic fluid, which is simulated by a Criminale-Ericksen-Filbey model, between two concentric rotating spheres is analyzed theoretically. This problem has wide applications in practice. The assessment of the performance of a polymerization reactor, for example, requires knowledge about the rheological properties of the reacting fluids and flow patterns around agitators. We show that the shear-thinning effects of both the viscosity and the elasticity of a fluid may lead to double-vortex flows. The origin of these vortices are discussed, and the effect of the relative sizes of the inner and the outer spheres on the vortex flows is investigated. The influence of the elasticity on the torque required to rotate the spheres is also evaluated. (C) 2002 Elsevier Science Ltd. All rights reserved.
\end{abstract}

Keywords: Viscoelastic fluid; Shear-thinning effects; Criminale-Ericksen-Filbey model; Concentric rotating spheres

\section{Introduction}

Studies of rotating fluid systems have been a very important part of fluid mechanics, since all fluid phenomena in practice involve rotation to a greater or lesser extent. The dynamics of rotating fluids, especially in rapidly rotating systems, are fascinating (Greenspan, 1968). Many unique features are observed in these systems that do not occur in non-rotating fluid environments. This is primarily due to the dominance of Coriolis forces acting on the motion of fluid in planes perpendicular to the rotational axis. Among various rotating fluid systems, the motion of a viscous fluid contained between two concentric rotating spheres is of special interest to researchers and design engineers because of its wide applications in various fields, for example, centrifuges, fluid gyroscopes (Pedlosky, 1969), and colloidal science (Endo \& Kousaka, 1996; Nakabayashi \& Tsuchida, 1995; Nakabayashi, Tsuchida, Takeo, \& Ono, 1995) . Although most of the theoretical analyses on the subject under consideration are based on Newtonian fluids, many potential applications in practice, however, involve non-Newtonian fluids (Bird, Armstrong, \& Hassager, 1977). The evaluation of the performance of a polymerization reactor, for instance,

\footnotetext{
${ }^{*}$ Corresponding author. Tel.: +886-2-2363-7448; fax: +886-22362-3040.

E-mail address:.jphsu@ccms.ntu.edu.tw (J.-P. Hsu).
}

requires a detailed understanding of the rheological properties of the reacting fluids and flow patterns around agitators (Ide \& White, 1974). The polymeric fluids involved are either in melt or solution form, and they exhibit elastic behavior in addition to the viscous behavior generally observed in Newtonian fluids. The study of the viscoelastic fluid contained between rotating spheres has direct application in this case, since a stirred-tank type reactor is typically used to process the fluids. Flow patterns and overall reactor performance are directly affected by the viscoelastic response of the fluid in a reactor. Bar-Yoseph and Kryzhanovski (1996) examined the breakdown of axisymmetric vortex for generalized Newtonian fluid contained between two rotating spheres. The classic Boussinesg problem is reconsidered for a Carreau fluid held between two concentric spheres at different temperatures. This is an improvement over the second order fluid (SOF) (Bird et al., 1977) used by Ide and White (1974) in that shear-thinning effect is included in the viscosity parameter expression. However, since the elasticity is neglected, the Carreau model is purely viscous. To combine the rheology merits of SOF and Carreau fluids the Criminale-Ericksen-Filbey equation (CEF) (Bird et al., 1977) is a natural generalization of either case. This model is exact for viscometric flows of elastic liquids, a nice property for special cases. It is also an extension of the well-known generalized Newtonian fluid (GNF) model. Therefore, the widely observed shear-thinning effect of a fluid, a crucial 
factor in the determination of the flow field under study, both in the sense of viscosity and elasticity can be nicely incorporated.

The flow of a Newtonian fluid between two concentric rotating spheres has received considerable attention in the literature. Most of the theoretical studies are of analytical nature in the early stages (Proudman, 1956; Munson \& Joseph, 1971). Only recently have numerical studies been undertaken on related problems. Greenspan (1975) used a finite difference method to solve the governing equations of the flow system. A more accurate fourth order central-difference discretization scheme was applied by Dennis and Quartapelle (1984) to solve the problem. Schultz, Schwengels, Pralhad, and Kocamustafaogullari (1991) investigated the non-isothermal case by adopting a second order finite difference scheme, and results for high Reynolds number were obtained. Essentially almost all the numerical methods used so far are based on a finite difference scheme. As compared to Newtonian fluids results available for viscoelastic fluids are very limited. Ide and White (1974), for example, analyzed the flow pattern around the agitator of a polymerization reactor for a polystyrene-styrene system. Experimental data were provided and compared with the theoretical predictions based on the SOF model. Again, a finite difference method was used in their numerical analysis. The rheological models adopted in the available studies for the behavior of a viscoelastic fluid have not, however, incorporated shear-thinning, a widely observed effect in practice (Bird et al., 1977). In other words, they represent at best the limiting case when this effect is absent. As pointed out previously, shear-thinning plays a significant role in the description of flow field, and a model which takes it into account is highly desirable.

In this study, the classical analysis for Newtonian fluids is extended to the case of viscoelastic fluids. For illustration, the CEF model is adopted to simulate the viscoelastic characteristics of these fluids. Because of the complicated situation encountered in the analysis, the stream function and vorticity formulation are the most commonly used approach to treat the governing equations of fluid flow. As a result, the highest order of the derivatives is fourth, which is higher than the second order derivatives normally encountered in the applications of the finite difference scheme. Here, we present the pseudo-spectral method as the discretization scheme for the partial differential equations (Lim \& Schowalter, 1987). It is best suited for globally smooth systems and calculation of high order derivatives with this scheme is straightforward. This method is a simplified version of the spectral method (Gottlieb \& Orszag, 1977; Gottlieb, Hussaini, \& Orszag, 1984). Compared with other numerical schemes, such as the finite difference method and the finite element method, the spectral method achieves higher accuracy with fewer nodal points, and therefore, smaller memory storage space is required. Moreover, the approximation error decreases exponentially with this approach, while only algebraically for the finite difference scheme, for example.

\section{Theory}

A schematic representation of the problem under consideration is illustrated in Fig. 1. A non-Newtonian fluid is confined between two concentric spheres. The radius of the inner and that of the outer spheres are $R_{i}$ and $R_{o}$, respectively. The inner and the outer spheres are rotating independently with angular velocity $\omega_{i}$ and $\omega_{o}$, respectively, around the $z$-axis. The spherical coordinates $(r, \theta, \phi)$ are chosen with its origin located at the center of the inner sphere. Let $v_{r}, v_{\theta}$, and $v_{\phi}$ be the components of the fluid velocity in $r, \theta$, and $\phi$ directions, respectively. The axisymmetric nature of the problem under consideration suggests that the dependent variables involved are independent of $\phi$, and therefore the computational domain reduces to a two-dimensional field $(r, \theta)$. The actual flow field, however, is three-dimensional, that is, $v_{\phi} \neq 0$, in general. Due to the symmetric structure of the system, representing its behavior in one of the four quadrants is sufficient. At steady state, the equations governing the flow field include the equation of motion, the continuity equation, and the constitutive equation. We have,

$$
\begin{aligned}
& \rho \underline{v}^{*} \bullet \nabla^{*} \underline{v}^{*}=-\nabla^{*} P^{*}-\nabla^{*} \underline{\underline{\tau}}^{*}, \\
& \nabla^{*} \bullet \underline{v}^{*}=0, \\
& \underline{\underline{\tau}}^{*}=-\eta^{*}\left(\dot{\gamma}^{*}\right) \underline{\underline{\hat{\gamma}^{*}}}-\left[\frac{1}{2} \Psi_{1}^{*}\left(\dot{\gamma}^{*}\right)+\Psi_{2}^{*}\left(\dot{\gamma}^{*}\right)\right]\left(\underline{\underline{\hat{\gamma}}}^{*} \bullet \underline{\underline{\hat{\gamma}}}^{*}\right) \\
& +\frac{1}{2} \Psi_{1}^{*}\left(\dot{\gamma}^{*}\right) \frac{\vartheta}{\vartheta t^{*}} \underline{\dot{\hat{\gamma}}}^{*}=\underline{\underline{\tau}}^{*}+\underline{\tau}^{*} p,
\end{aligned}
$$

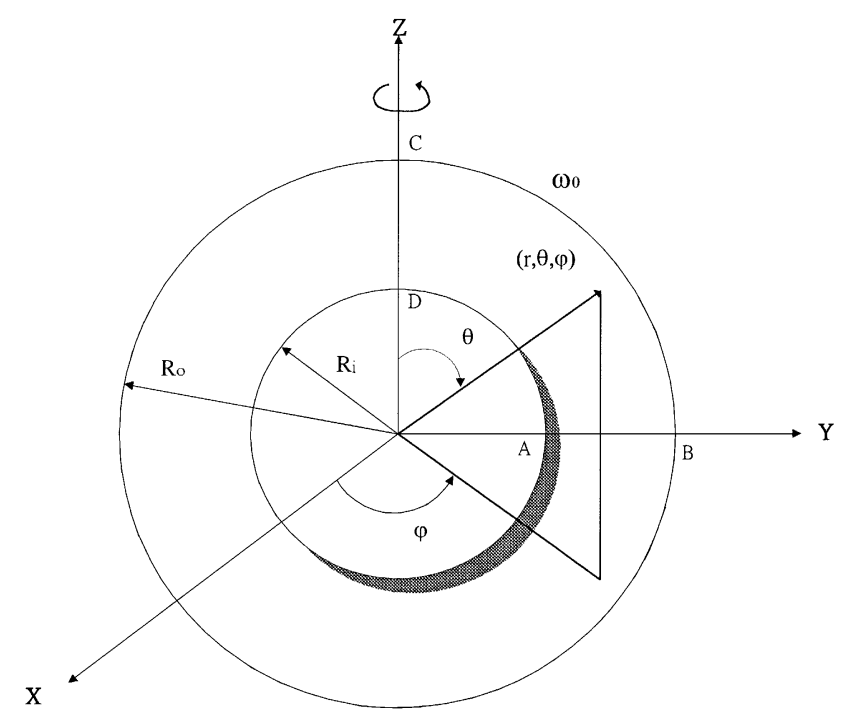

Fig. 1. Schematic representation of the problem under consideration. A non-Newtonian fluid is confined between two concentric spheres. The radius of the inner and that of the outer spheres are $R_{i}$ and $R_{o}$, respectively. The inner and the outer spheres are rotating independently with angular velocity $\omega_{i}$ and $\omega_{o}$, respectively, around the $z$-axis. 
where

$$
\begin{aligned}
& \underline{\tau}_{s}^{*}=-\eta^{*}\left(\dot{\gamma}^{*}\right) \underline{\underline{\hat{\gamma}}}^{*}, \\
& \stackrel{\tau^{*}}{=} p=-\left[\frac{1}{2} \Psi_{1}^{*}\left(\dot{\gamma}^{*}\right)+\Psi_{2}^{*}\left(\dot{\gamma}^{*}\right)\right]\left(\underline{\underline{\hat{\gamma}}}^{*} \bullet \underline{\underline{\hat{\gamma}}}^{*}\right) \\
& +\frac{1}{2} \Psi_{1}^{*}\left(\dot{\gamma}^{*}\right) \frac{\vartheta}{\vartheta t^{*}} \underline{\dot{\gamma}}^{*}
\end{aligned}
$$

The definitions of the symbols in Eqs. (1)-(3) are given in the Notation. The stress tensor $\tau^{*}$ is decomposed into to $\underline{\tau}^{*}$ and $\underline{\tau}_{p}^{*}$ for convenience. The former corresponding to the purely viscous contribution from the GNF model and the latter corresponds to the contribution which arises from the elastic nature of the fluid. Apparently, the GNF model can be recovered as the special case of the present CEF model by ignoring the elasticity effect. The asterisk refers to a scaled quantity. Eq. (3) is the Criminale-Ericksen-Filbey (CEF) equation, where $\underline{\tau}_{s}^{*}$ represents the contribution from GNF, and $\stackrel{\tau}{=}_{p}^{*}$ is that from the elastic nature of the fluid. $\vartheta / \vartheta t^{*}$ is the corotational time derivative or Jaumann derivative. The material functions $\eta^{*}, \Psi_{1}^{*}$, and $\Psi_{2}^{*}$ are related to the shear rate $\dot{\gamma}^{*}$. They are described by the Carreau model

$\eta^{*}=\eta_{0}^{*}\left[1+\left(\lambda^{*} \dot{\gamma}^{*}\right)^{2}\right]^{(n-1) / 2}$,

$\Psi_{1}^{*}=\Psi_{1,0}^{*}\left[1+\left(\lambda_{p}^{*} \dot{\gamma}^{*}\right)^{2}\right]^{(p-2) / 2}$,

$\Psi_{2}^{*}=\Psi_{2,0}^{*}\left[1+\left(\lambda_{q}^{*} \dot{\gamma}^{*}\right)^{2}\right]^{(q-2) / 2}$,

where $\eta_{0}^{*}, \Psi_{1,0}^{*}$, and $\Psi_{2,0}^{*}$ are, respectively, the values of the material functions $\eta^{*}, \Psi_{1}^{*}$, and $\Psi_{2}^{*}$ at zero shear rate. The time constants $\lambda^{*}, \lambda_{p}^{*}$, and $\lambda_{q}^{*}$ are associated with $\eta^{*}, \Psi_{1}^{*}$, and $\Psi_{2}^{*}$, respectively. The corotational time derivative in Eq. (3) at steady state is defined by

$\frac{\vartheta}{\vartheta t^{*}} \underline{\bar{\gamma}}^{*}=\underline{v}^{*} \bullet \nabla^{*} \underline{\underline{\dot{\gamma}}}^{*}+\frac{1}{2}\left[\left(\underline{\underline{\omega}}^{*} \bullet \underline{\underline{\bar{\gamma}}}^{*}\right)-\left(\underline{\underline{\bar{\gamma}}}^{*} \bullet \underline{\underline{\omega}}^{*}\right)\right]$.

Here, the effect of gravitational force is neglected. We define $r^{*}=R_{s} r, t^{*}=t_{s} t, v^{*}=R_{s} v / t_{s}, \underline{\underline{\tau}}{ }^{*}=\eta_{0} \underline{\underline{\tau}} / t_{s}, P^{*}=$ $\eta_{0} P / t_{s}, \underline{\overline{\hat{\gamma}}}^{*}=\dot{\bar{\gamma}} / t_{s}, \underline{\underline{\omega}}^{*}=\underline{\underline{\omega}} / t_{s}, \lambda^{*}=t_{s} \lambda, \lambda_{p}^{*}=t_{s} \lambda_{p}, \lambda_{q}^{*}=t_{s} \lambda_{q}$, $\eta^{*}=\eta_{0} \overline{\bar{\eta}}, \quad \Psi_{1}^{\bar{*}}=2 \eta_{0} \Psi_{1} / \omega_{s}, \quad$ and $\Psi_{2}^{*}=\eta_{0} \Psi_{2} / \omega_{s}$, where $\omega_{s}=\max \left\{\omega_{i}, \omega_{o}\right\}$. On the basis of these variables Eqs. (1)-(6) become, respectively,

$R e \underline{v} \bullet \nabla \underline{v}=-\nabla P-\nabla \bullet \underline{\underline{\tau}}$,

$\nabla \bullet \underline{v}=0$,

$\underline{\underline{\tau}}=-\eta(\dot{\gamma}) \underline{\underline{\bar{\gamma}}}-\left(\Psi_{1}+\Psi_{2}\right)(\underline{\underline{\bar{\gamma}}} \bullet \underline{\underline{\bar{\gamma}}})+\Psi_{1} \frac{\vartheta}{\vartheta t} \stackrel{\dot{\bar{\gamma}}}{=}$

$\eta=\left[1+(\lambda \dot{\gamma})^{2}\right]^{(n-1) / 2}$,

$\Psi_{1}=D e\left[1+\left(\lambda_{p} \dot{\gamma}\right)^{2}\right]^{(p-2) / 2}$

$\Psi_{2}=L e\left[1+\left(\lambda_{q} \dot{\gamma}\right)^{2}\right]^{(q-2) / 2}$.
In these expressions, the Reynolds number $R e$, the Deborah number $D e$, and the Lewis number Le are defined, respectively, by $R e=\rho R_{o}^{2} \omega_{s} / \eta_{0}, D e=\Psi_{1,0}^{*} \omega_{s} / 2 \eta_{0}$, and $L e=\Psi_{2,0}^{*} \omega_{s} / \eta_{0}$. Therefore, the momentum equation has the form

$$
\begin{aligned}
& \operatorname{Re} \underline{v} \bullet \nabla \underline{v}=-\nabla p-\nabla \bullet\left(\underline{\tau}_{s}+\underline{\underline{\tau}}_{p}\right) \\
& =-\nabla p+\eta \nabla^{2} \underline{v}+\nabla \eta \bullet \underline{\underline{\bar{\gamma}}} \\
& +\nabla\left(\Psi_{1}+\Psi_{2}\right) \bullet(\underline{\underline{\bar{\gamma}}} \bullet \underline{\underline{\bar{\gamma}}}) \\
& +\left(\Psi_{1}+\Psi_{2}\right) \nabla \bullet(\underline{\underline{\bar{\gamma}}} \bullet \underline{\overline{\bar{\gamma}}}) \\
& -\left(\nabla \Psi_{1}\right) \bullet \frac{\vartheta}{\vartheta t} \stackrel{\dot{\bar{\gamma}}}{=}-\Psi_{1} \nabla \bullet \frac{\vartheta}{\vartheta t} \dot{\bar{\gamma}} .
\end{aligned}
$$

Conventional no-slip boundary conditions are assumed on the surfaces of the spheres. We have

$v_{r}=v_{\theta}=0$ on surfaces $\mathrm{AD}$ and $\mathrm{BC}$,

$v_{\phi}=\left(\frac{\omega_{i}}{\omega_{s}}\right)\left(\frac{R_{i}}{R_{o}}\right) \sin \theta$ on surface $\mathrm{AD}$,

$v_{\phi}=\left(\frac{\omega_{o}}{\omega_{s}}\right) \sin \theta$ on surface BC.

Since the computational domain is two-dimensional, advantages can be taken to reduce the number of unknown variables of velocity by introducing the scaled stream function $\psi$. Note that the continuity equation is satisfied automatically with this approach. In terms of $\psi, v_{r}$ and $v_{\theta}$ can be expressed by

$v_{r}=\frac{\partial \psi / \partial \theta}{r^{2} \sin \theta}$

$v_{\theta}=\frac{\partial \psi / \partial r}{r \sin \theta}$

where the dimensionless stream function is scaled by $R_{o}^{3} \omega_{s}$. The pressure term in Eq. (14) can be removed by taking curl, which is equivalent to replacing the original velocity terms by introducing the scaled vorticity formulation $\Omega$ :

$v_{\phi}=\frac{\Omega}{r \sin \theta}$

which can be scaled by $R_{o}^{2} \omega_{s}$. As mentioned earlier, the flow system under study is axisymmetric, therefore all quantities are $\phi$-independent. Substituting Eqs. (18)-(20) into Eq. (14), we obtain

$$
\begin{aligned}
& \eta D^{2} \Omega+\frac{R e}{r^{2} \sin \theta}\left(\frac{\partial \psi}{\partial r} \frac{\partial \Omega}{\partial \theta}-\frac{\partial \psi}{\partial \theta} \frac{\partial \Omega}{\partial r}\right) \\
& =-r \sin \theta\left[\frac{\partial \eta}{\partial r} \dot{\gamma}_{r \phi}+\frac{1}{r} \frac{\partial \eta}{\partial \theta} \dot{\gamma}_{\theta \phi}-\left(\nabla \bullet \underline{\underline{\tau}}_{p}\right)_{\phi}\right]
\end{aligned}
$$


and

$$
\begin{aligned}
\eta D^{4} \psi & +\operatorname{Re}\left[\frac{2 \Omega}{r^{3} \sin ^{2} \theta}\left(\sin \theta \frac{\partial \Omega}{\partial \theta}-r \cos \theta \frac{\partial \Omega}{\partial r}\right)\right. \\
+ & \frac{1}{r^{2} \sin \theta}\left(\frac{\partial \psi}{\partial r} \frac{\partial}{\partial \theta} D^{2} \psi-\frac{\partial \psi}{\partial \theta} \frac{\partial}{\partial r} D^{2} \psi\right) \\
& \left.+\frac{2 D^{2} \psi}{r^{3} \sin ^{2} \theta}\left(\sin \theta \frac{\partial \psi}{\partial \theta}-r \cos \theta \frac{\partial \psi}{\partial r}\right)\right] \\
= & -\sin \theta\left\{\left(\nabla^{2} \underline{v}\right)_{r} \frac{\partial \eta}{\partial \theta}-r\left(\nabla^{2} \underline{v}\right)_{\theta} \frac{\partial \eta}{\partial r}\right. \\
+ & \frac{\partial}{\partial \theta}(\nabla \eta \bullet \stackrel{\dot{\bar{\gamma}}}{=})_{r}-\frac{\partial}{\partial r}\left[r(\nabla \eta \bullet \stackrel{\dot{\bar{\gamma}}}{=})_{\theta}\right] \\
& \left.-\frac{\partial}{\partial \theta}(\nabla \bullet \stackrel{\tau}{=})_{r}+\frac{\partial}{\partial r}\left[r(\nabla \bullet \stackrel{\tau}{=})_{\theta}\right]\right\},
\end{aligned}
$$

where

$D^{2}=\frac{\partial^{2}}{\partial r^{2}}+\frac{1}{r^{2}} \frac{\partial^{2}}{\partial \theta^{2}}-\frac{\cot \theta}{r^{2}} \frac{\partial}{\partial \theta}$

with $D^{4}=D^{2}\left(D^{2}\right)$. The corresponding scaled boundary conditions are

$\psi=\frac{\partial \psi}{\partial r}=0$ on surfaces $\mathrm{AD}$ and $\mathrm{BC}$,

$\psi=\Omega=0$ on surface $\mathrm{CD}$,

$\Omega=\left(\frac{\omega_{i}}{\omega_{s}}\right)\left(\frac{R_{i}}{R_{o}}\right)^{2} \sin ^{2} \theta$ on surface AD,

$\Omega=\left(\frac{\omega_{o}}{\omega_{s}}\right) \sin ^{2} \theta$ on surface BC,

$\psi=\frac{\partial \Omega}{\partial \theta}=0$ on surface AB.

Eqs. (21) and (22) and the associated boundary conditions, Eqs. (24)-(28), constitute a well-posed problem which is solved by the pseudo-spectral method based on Chebyshev polynomials. This method is readily applicable for the present problem, and it has several advantages such as a high order of convergence and the convergent properties independent of the boundary conditions (Nakabayashi et al., 1995; Gottlieb \& Orszag, 1977). In our case, the computational domain is $(r, \theta)$. For an unknown function $f(r, \theta)$, we assume that its $N \times M$ th-order approximation, $f_{N M}(r, \theta)$, can be expressed as

$f_{N M}(r, \theta)=\sum_{i=0}^{N} \sum_{j=0}^{M} f_{N M}\left(r_{i}, \theta_{j}\right) g_{i}(r) g_{j}(\theta)$,

where $f_{N M}\left(r_{i}, \theta_{j}\right)$ is the value of $f_{N M}$ at the $k$ th $(k=$ $(N-1) i+j)$ collocation point. The interpolation polynomials $g_{i}(r)$ and $g_{j}(\theta)$ depend on the locations of the collocation points, which are determined by mapping the computational domain onto the region $[-1,1] \times[-1,1]$ by

$r=\frac{R_{o}-R_{i}}{2} y+\frac{R_{o}+R_{i}}{2}$,

$\theta=\frac{\pi}{4}(x+1)$.

The $N+1$ interpolation points in the interval $[-1,1]$ are chosen to be the extreme values of an $N$ th order Chebyshev polynomial $\left(T_{N}(y)\right)$

$y_{j}=\cos \left(\frac{\pi j}{N}\right), \quad j=0,1, \ldots, N$.

The corresponding interpolation polynomial $g_{j}(y)$ is

$g_{j}(y)=\frac{(-1)^{j+1}\left(1-y^{2}\right)\left(\mathrm{d} T_{N} / \mathrm{d} y\right)}{c_{j} N^{2}\left(y-y_{j}\right)}, \quad j=0,1, \ldots, N$

where $c_{j}$ is defined by

$c_{j}= \begin{cases}2, & j=0, N, \\ 1, & 1 \leqslant j \leqslant N-1 .\end{cases}$

Partial derivatives of $f_{N M}(r, \theta)$ can be obtained by differentiating Eq. (33). The interpolation polynomials and collocation points in the $\theta$-direction can be determined in a similar manner.

Applying the above discretization scheme to the governing equations yields a set of non-linear algebraic equations of stream function $\psi$ and vorticity function $\Omega$ which is solved by a Newton-Raphson iteration scheme. Double precision is used throughout the computation. To ensure that the mesh used is fine enough so that the solution of the system of algebraic equations converges to the original system of partial differential equations, a test of mesh refinement is carried out. Three different mesh patterns, which include $10 \times 10,12 \times 12$, and $14 \times 14$ nodal points, were examined to guarantee the convergence of accuracy. The results of calculation for the last two mesh patterns essentially coincide with each other, indicating that the last mesh pattern is fine enough. Having assured its suitability, all of the calculations presented in this study were based on the last mesh pattern, which is presented in Fig. 2.

\section{Results and discussion}

The behavior of the system under consideration is examined through numerical simulation. The parameters used in the material functions are $n=0.2, \lambda=1.41, \lambda_{p}=\lambda_{q}=1.41$, $p=q=0.54$, and $\Psi_{2}=-0.1 \Psi_{1}$. These values are based partially on the limited data available in the literature (Bird et al., 1977). The flow field is thus a function of Deborah number only. For illustration, we consider the case $\omega_{i}=1.0$ and $\omega_{o}=0$, which simulates a stirred-tank-type polymerization reactor. To test the effect of elasticity, we focus on the creeping flow situation, that is, $R e=0$; the inertia force 

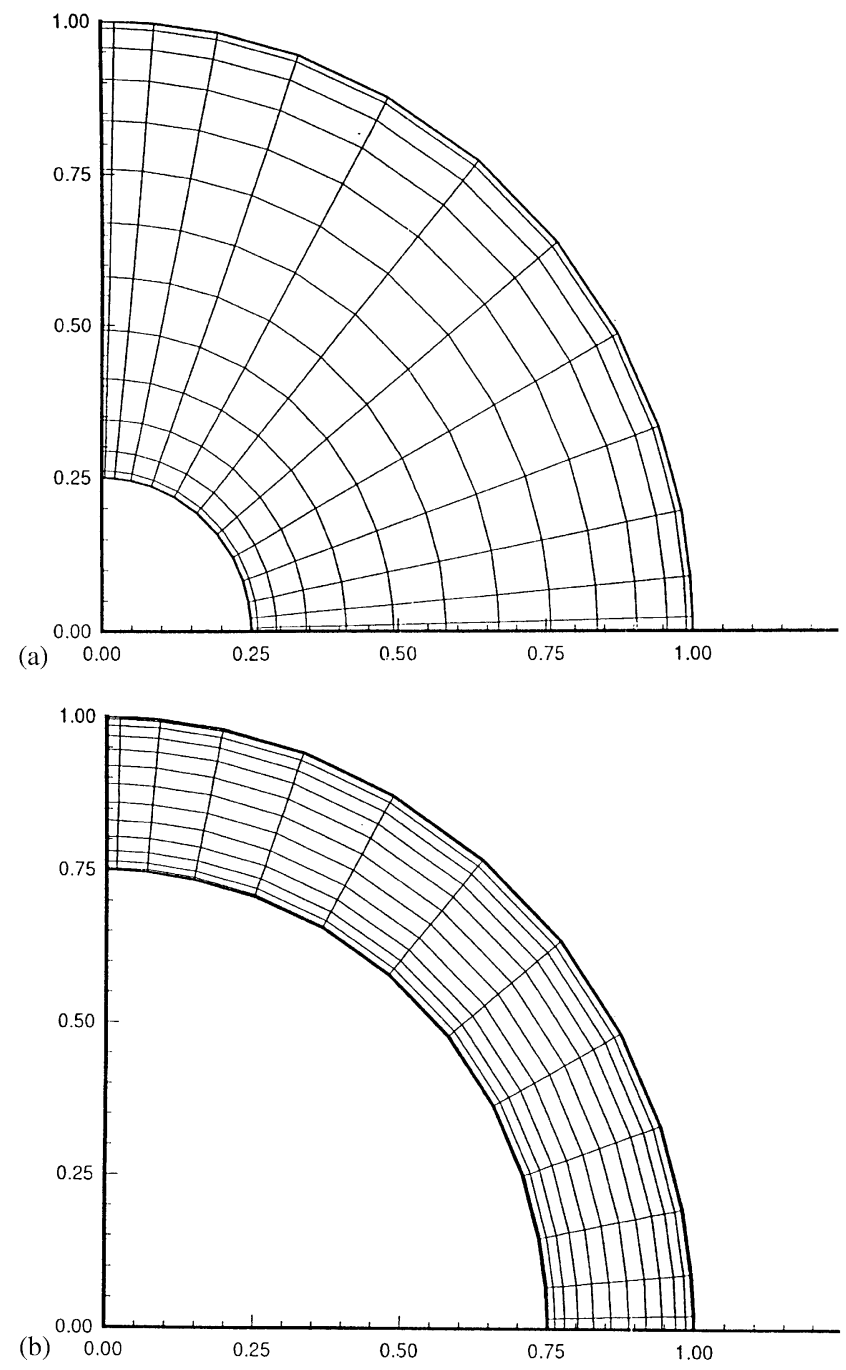

Fig. 2. Mesh pattern adopted with a total of $14 \times 14$ nodal points with $R_{o}=1$. (a) $R_{i}=0.25$, (b) $R_{i}=0.75$.

is absent in this situation. An exact analytical solution is available for this system if the fluid under consideration is Newtonian (Happel \& Brenner, 1983). The result based on our numerical algorithm agrees excellently with this exact solution. Quantitatively, the flow field behaves like a rigid body moving along the $\phi$-direction for the Newtonian case. No secondary flow in the $r-\theta$ plane is observed.

Fig. 3 illustrates the contours of stream function $\psi$ for the case of Newtonian fluid with $R e=1.0$. As can be seen from this figure, the introduction of inertia force, which is represented by a non-zero $R e$, induces a counterclockwise secondary flow in the $r-\theta$ plane; a single vortex flow occupies the entire first quadrant. The counterclockwise flow arises from the fact that the centrifugal force is greater near the equatorial area and the fluid is spinned off toward the outer sphere.

Figs. 4-7 show the contours of stream function $\psi$ for the case of CEF fluid with $R e=0$ at various $D e$. These figures reveal that when elasticity is introduced, which is repre-

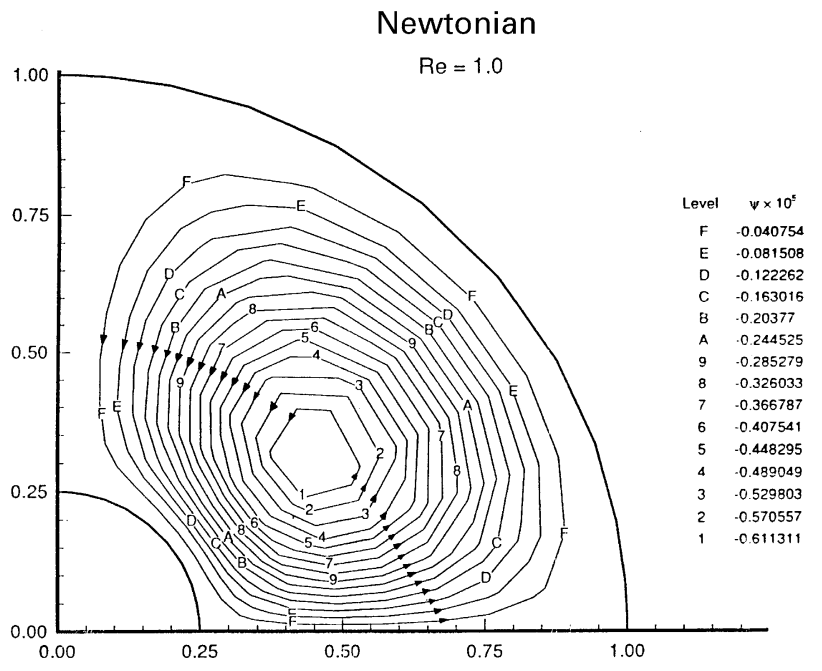

Fig. 3. Contours of stream function $\psi\left(\times 10^{5}\right)$ for the case of Newtonian fluid with $R e=1.0$ and $R_{i}=0.25$. Key: $R_{o}=1.0, \omega_{i}=1$, $\omega_{o}=0, n=0.2, \lambda=1.41, \lambda_{p}=\lambda_{q}=1.41, p=q=0.54$, and $\Psi_{2}=-0.1 \Psi_{1}$.

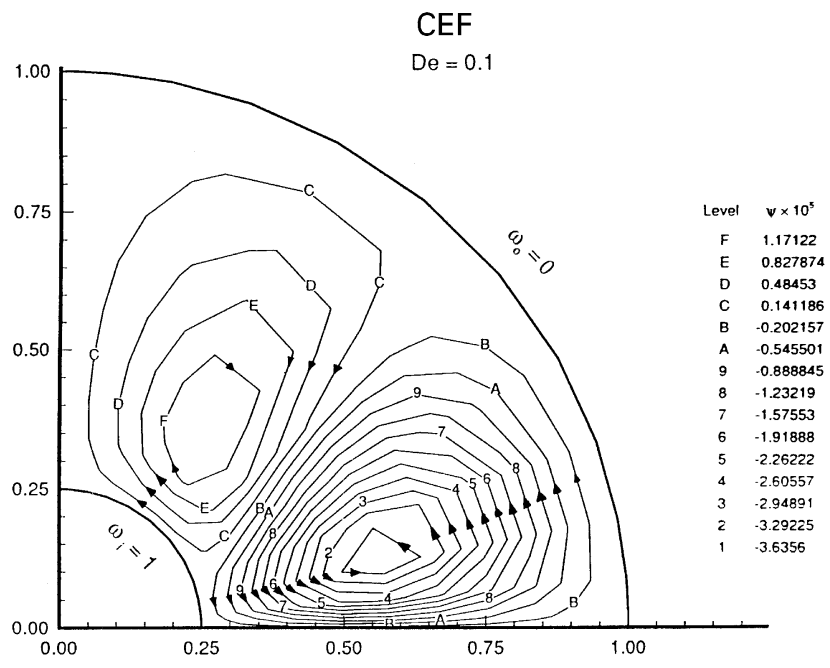

Fig. 4. Contours of stream function $\psi\left(\times 10^{5}\right)$ for the case of CEF fluid with $R e=0, D e=0.1$ and $R_{i}=0.25$. Key: same as Fig. 3 .

sented by a non-zero $D e$, a secondary flow appears although $R e=0$. Note that the upper vortex near the north-pole area is clockwise, while the inertia-induced secondary vortex flow is counterclockwise in the previous Newtonianaise. Obviously, the secondly flow observed here is induced by elasticity, since no inertia effect is involved $(R e=0)$. Fig. 5 shows that if $D e$ is increased, which implies that the effect of elasticity is enhanced, both the strength and the area occupied by the upper vortex increase. As illustrated in Fig. 6, this trend continues until De reaches about 0.6. In this case, the upper vortex dominates the flow field, and the lower vortex disappears completely. Fig. 7 shows that a further increase in De has the effect of increasing the strength of the clockwise vortex, as expected. The complete disappearance of the second vortex is one of the fascinating phenomena observed in the viscoelastic flow considered. 


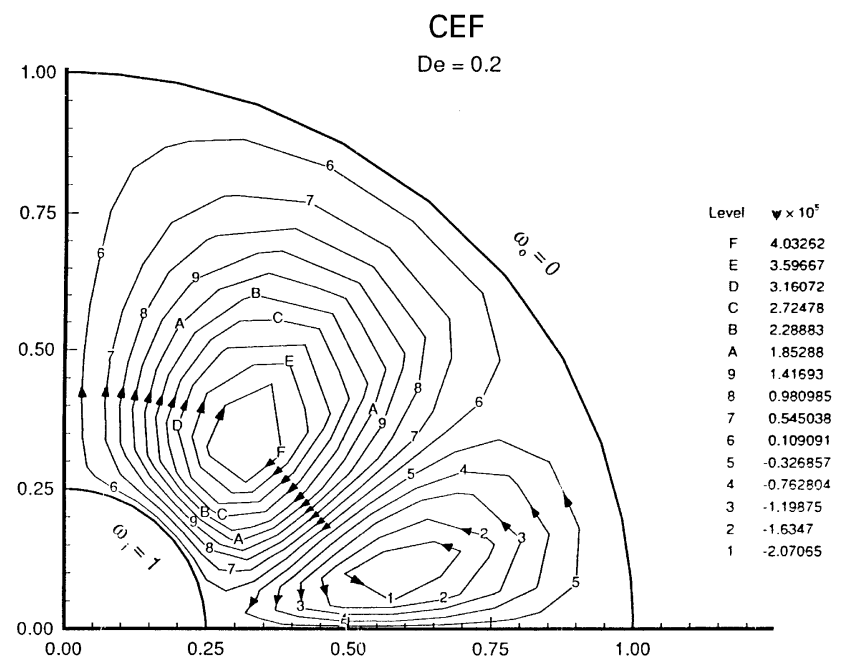

Fig. 5. Contours of stream function $\psi\left(\times 10^{5}\right)$ for the case of CEF fluid with $R e=0, D e=0.2$ and $R_{i}=0.25$. Key: same as Fig. 3 .

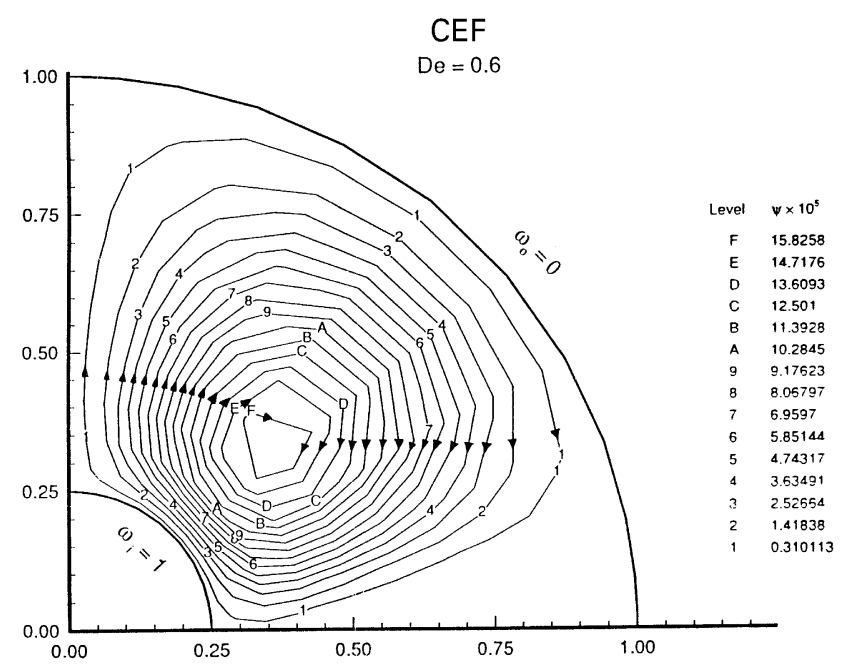

Fig. 6. Contours of stream function $\psi\left(\times 10^{5}\right)$ for the case of CEF fluid with $R e=0, D e=0.6$ and $R_{i}=0.25$. Key: same as Fig. 3 .

We also present the contours of stream function $\psi$ for the case of CEF fluid with $R e=0$ for other combinations of $D e$ and $R_{i}$. It is interesting to note that if the radius of the inner sphere $R_{i}$ is increased to 0.75 , only one vortex exists with clockwise orientation for $D e$ all the way up to 1.0, as shown in Figs. 8 and 9. A careful reviewing of the corresponding velocity vector plots reveals that the strength of the vortex is larger in the upper area, especially near the surface of the inner sphere. However, it never breaks into two separate vortices throughout the range of $D e$ examined. Therefore, we conclude that the effect of elasticity on the secondary flow depends strongly on the relative magnitudes of the inner and the outer spheres.

One of the important physical properties available from the basis flow discussed above is the torque required to rotate the sphere at a given rate. The scaled torque on the

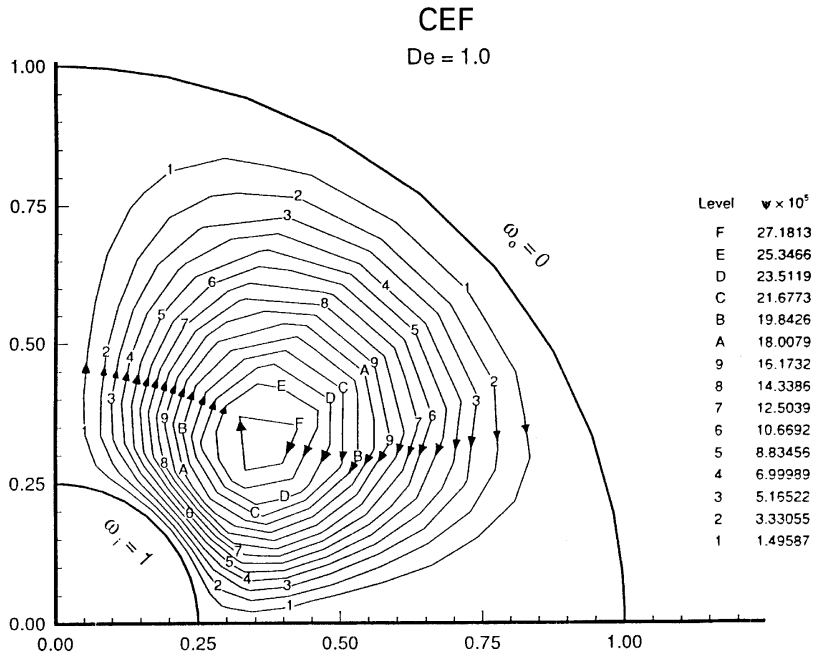

Fig. 7. Contours of stream function $\psi\left(\times 10^{5}\right)$ for the case of CEF fluid with $R e=0, D e=1.0$ and $R_{i}=0.25$. Key: same as Fig. 3 .

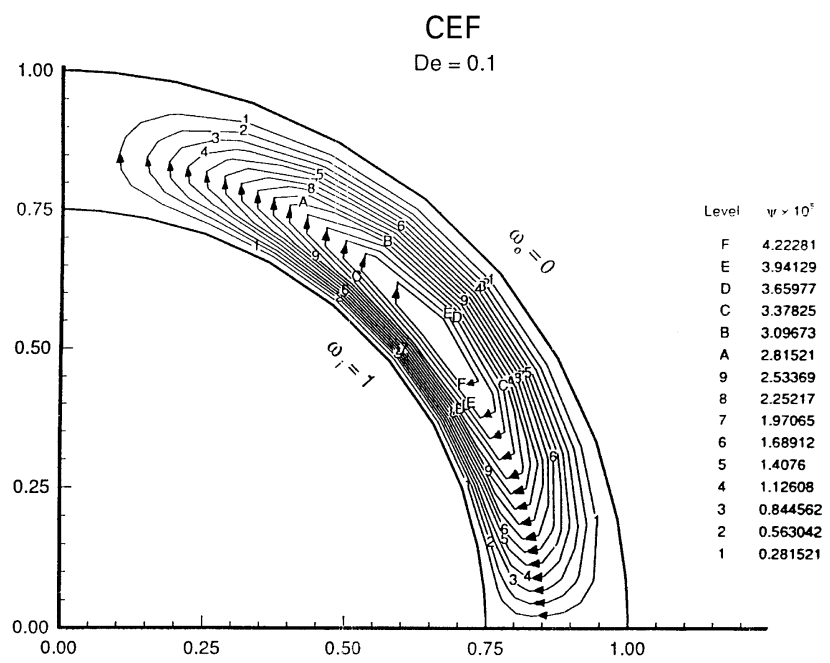

Fig. 8. Contours of stream function $\psi\left(\times 10^{5}\right)$ for the case of CEF fluid with $R e=0, D e=0.1$ and $R_{i}=0.75$. Key: same as Fig. 3 .

inner sphere, $T_{i}^{*}$, and that on the outer sphere, $T_{o}^{*}$, can be evaluated, respectively, by

$$
\begin{aligned}
& T_{i}^{*}=-\left.4 \pi R_{i}^{3} \int_{0}^{\pi / 2} \tau_{r \phi}^{*}\right|_{r=R_{i}} \sin ^{2} \theta \mathrm{d} \theta, \\
& T_{o}^{*}=\left.4 \pi R_{o}^{3} \int_{0}^{\pi / 2} \tau_{r \phi}^{*}\right|_{r=R_{o}} \sin ^{2} \theta \mathrm{d} \theta,
\end{aligned}
$$

where the scaled torque $\tau_{r \phi}^{*}$ is the $r \phi$-component of the scaled stress tensor which is made non-dimensional by the characteristic torque, $8 \pi \eta_{0}^{*} \omega_{s} R_{o}^{3} / 3$. Note that $T_{i}^{*}$ and $T_{o}^{*}$ should have the same magnitude, but of opposite signs. This provides an additional check of the accuracy of the results calculated. The torques acting on the inner and outer spheres for two different $R_{i}$ are summarized in Tables 1 and 2. As can be seen from these tables, the consistency of the torques on the inner and outer spheres 


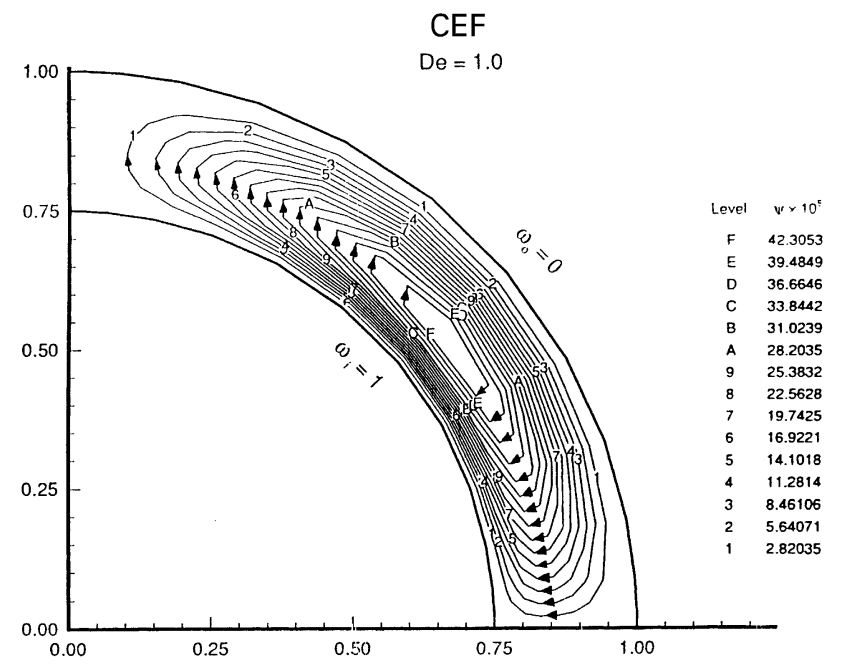

Fig. 9. Contours of stream function $\psi\left(\times 10^{5}\right)$ for the case of CEF fluid with $R e=0, D e=1.0$ and $R_{i}=0.75$. Key: same as Fig. 3 .

Table 1

Calculated values of the torque on the inner sphere, $T_{i}$, and that on the outer sphere, $T_{o}$, for various values of Deborah number for the case $R e=0, R_{i}=0.25, R_{o}=1, \omega_{i}=1, \omega_{o}=0, n=0.2, \lambda=1.41, \lambda_{p}=\lambda_{q}=$ $1.41, p=q=0.54$, and $\Psi_{2}=-0.1 \Psi_{1}$

\begin{tabular}{llll}
\hline De & $T_{o}^{*}$ & $T_{i}^{*}$ & Deviation (\%) \\
\hline 0.1 & -0.02135 & 0.02124 & 0.2583 \\
0.2 & -0.02130 & 0.02123 & 0.1646 \\
0.3 & -0.02124 & 0.02123 & 0.0235 \\
0.4 & -0.02119 & 0.02122 & 0.0707 \\
0.5 & -0.02112 & 0.02121 & 0.2126 \\
0.6 & -0.02107 & 0.02120 & 0.3075 \\
0.7 & -0.02101 & 0.02118 & 0.4029 \\
0.8 & -0.02097 & 0.02116 & 0.4510 \\
0.9 & -0.02090 & 0.02114 & 0.5709 \\
1.0 & -0.02083 & 0.02112 & 0.6913 \\
\hline
\end{tabular}

Table 2

Calculated values of the torque on the inner sphere, $T_{i}$, and that on the outer sphere, $T_{o}$, for various values of Deborah number for the case $R e=0, R_{i}=0.75, R_{o}=1, \omega_{i}=1, \omega_{o}=0, n=0.2, \lambda=1.41, \lambda_{p}=\lambda_{q}=$ 1.41, $p=q=0.54$, and $\Psi_{2}=-0.1 \Psi_{1}$

\begin{tabular}{llll}
\hline De & $T_{o}^{*}$ & $T_{i}^{*}$ & Deviation $(\%)$ \\
\hline 0.1 & -0.60728 & 0.60731 & 0.0025 \\
0.2 & -0.60715 & 0.60728 & 0.0107 \\
0.3 & -0.60694 & 0.60722 & 0.0231 \\
0.4 & -0.60663 & 0.60714 & 0.0420 \\
0.5 & -0.60623 & 0.60704 & 0.0668 \\
0.6 & -0.60573 & 0.60692 & 0.0981 \\
0.7 & -0.60513 & 0.60678 & 0.1361 \\
0.8 & -0.60433 & 0.60662 & 0.1891 \\
0.9 & -0.60360 & 0.60644 & 0.2347 \\
1.0 & -0.60265 & 0.60625 & 0.2978 \\
\hline
\end{tabular}

is satisfactory. Tables 1 and 2 suggest that $\left|T_{o}^{*}\right|$ or $T_{i}^{*}$ decreases slightly with the increase in $D e$.

\section{Conclusion}

The flow of a viscoelastic fluid between concentric rotating spheres were analyzed theoretically. The Criminale-Ericksen-Filbey (CEF) equation, which takes the shear-thinning effect into account, is adopted to describe the rheological behavior of the fluid. The results of numerical simulation reveal that if the inner sphere is small, double vortices secondary flow appear in the first quadrant of the flow region when Deborah number is small. This secondary flow is induced by the elasticity. The flow orientation of the upper vortex near the north-pole area is clockwise, while that of the lower vortex is counterclockwise. As Deborah number is further increased, which implies the effect of elasticity is enhanced, the strength and area occupied by the upper vortex increases accordingly. The lower vortex disappears if Deborah number is sufficiently large. If the inner sphere becomes large, only one vortex flow with clockwise orientation is observed for the whole range of Deborah number considered. The absolute value of the torque on the spheres is found to decrease slightly with the increase in Deborah number.

\section{Notation}

De Deborah number $\left(=\Psi_{1,0}^{*} \omega_{s}^{*} / 2 \eta_{0}^{*}\right)$

Le Lewis number $\left(\Psi_{2,0}^{*} \omega_{s}^{*} / \eta_{0}^{*}\right)$

$n \quad$ power-law exponent of $\eta$

$p \quad$ power-law exponent of $\Psi_{1}$

$P \quad$ hydrodynamic pressure

$q \quad$ power-law exponent of $\Psi_{2}$

$r \quad$ radial coordinate in spherical coordinates

$R \quad$ radius

Re Reynolds number $\left(=\rho^{*} R_{o}^{2} \omega_{s}^{*} / \eta_{0}^{*}\right)$

$T$ torque required to rotate the spheres at a given rate

$v \quad$ velocity

\section{Greek letters}

$\dot{\gamma} \quad$ magnitude of the rate of deformation tensor

$\eta \quad$ apparent viscosity

$\eta_{0} \quad$ zero-shear-rate viscosity

$\theta \quad$ angle measured downwards from the $z$-axis in spherical coordinates

$\lambda \quad$ time constant for viscosity

$\lambda_{p} \quad$ time constant for $\Psi_{1}$

$\lambda_{q} \quad$ time constant for $\Psi_{2}$

$\rho \quad$ fluid density

$\tau \quad$ magnitude of $\underline{\underline{\tau}}$

$\phi \quad$ angle measured around the $z$-axis in spherical coordinates

$\psi \quad$ stream function

$\Psi_{1} \quad$ primary normal stress coefficient

$\Psi_{2} \quad$ secondary normal stress coefficient

$\Psi_{1,0} \quad \Psi_{1}$ at zero shear rate 


$$
\begin{array}{ll}
\Psi_{2,0} & \Psi_{2} \text { at zero shear rate } \\
\omega & \text { angular velocity of sphere } \\
\Omega & \text { vorticity function }
\end{array}
$$

\section{Subscripts}

$i \quad$ inner sphere

$o \quad$ outer sphere

$s \quad$ characteristic quantity

\section{Superscripts}

\section{* $\quad$ scaled quantity}

$\mathrm{T} \quad$ matrix transpose

\section{Mathematical symbols}

$\begin{array}{ll}\nabla & \text { gradient } \\ \nabla \bullet & \text { divergence } \\ \nabla^{2} & \text { Laplacian }(=\nabla \bullet \nabla)\end{array}$

$\vartheta / \vartheta t \quad$ corotational time derivative or Jaumann derivative

$\mathrm{D} / \mathrm{D} t \quad$ substantial derivative $\left(=\frac{\partial}{\partial t}+v \bullet \nabla\right)$

- inner product

: double dot multiplication

$D^{2} \quad$ differential operator $\left(=\frac{\partial^{2}}{\partial r^{2}}+\frac{1}{r^{2}} \frac{\partial^{2}}{\partial \theta^{2}}-\frac{1}{r^{2}} \cot \theta \frac{\partial}{\partial \theta}\right)$

\section{Underline}

$\begin{array}{ll}\underline{v} & \begin{array}{l}\text { velocity vector } \\ \underline{\underline{\tau}}\end{array} \\ \begin{array}{l}\text { part of total stress tensor that vanishes at equilib- } \\ \text { rium }\end{array} \\ \underline{\underline{\underline{\tau}}} s & \text { GNF part of } \underline{\bar{\tau}} \\ \underline{\underline{\tau}} p & \text { elastic part of } \underline{\underline{\tau}} \\ \underline{\underline{\gamma}} & \text { rate of deformation tensor } \\ \underline{\underline{\omega}} & \text { vorticity tensor }\end{array}$

\section{Acknowledgements}

This work is supported by the National Science Council of the Republic of China.

\section{References}

Bar-Yoseph, P. Z., \& Kryzhanovski, Yu. (1996). Axisymmetric vortex breakdown for generalized Newtonian fluid contained between rotating spheres. Journal of Non-Newtonian Fluid Mechanics, 66, 145.
Bird, R.B., Armstrong, R.C., \& Hassager, O. (1977). Dynamics of polymeric liquids, Vol. 1 (2nd ed.) New York: Wiley.

Dennis, S. C. R., \& Quartapelle, L. (1984). Finite difference solution to the flow between two rotating spheres. Computers \& Fluids, 12, 77.

Endo, Y., \& Kousaka, Y. (1996). Dispersion mechanism of coagulated particles in liquid flow. Colloids Surfaces A, 109, 109.

Gottlieb, D., Hussaini, M. Y., \& Orszag, S. A. (1984). Spectral methods for partial differential equations. Philadelphia, PA: SIAM.

Gottlieb, D., \& Orszag, S.A. (1977). Numerical analysis of spectral methods: Theory and application. Philadelphia, PA: SIAM.

Greenspan, D. (1975). Numerical studies of steady, viscous, incompressible flow between two rotating spheres. Computers \& Fluids, 3, 69.

Greenspan, H. P. (1968). The theory of rotating fluids. New York: Cambridge University Press.

Happel, J., \& Brenner, H. (1983). Low Reynolds number hydrodynamics: With special application to particulate media. New York: Nijhoff.

Ide, Y., \& White, J. L. (1974). Rheological phenomena in polymerization reactors: Rheological properties and flow patterns around agitators in polystyrene-styrene solution. Journal of Applied Polymer Science, 18, 2997.

Lim, F. J., \& Schowalter, W. R. (1987). Pseudo-spectral analysis of the stability of pressure-driven flow of a giesekus fluid between parallel planes. Journal of Non-Newtonian Fluid Mechanics, 26, 135.

Munson, B. R., \& Joseph, D. D. (1971). Viscous in compressible flow between concentric rotating sphere. Part 1 basic flow. Journal of Fluid Mechanics, 49, 289.

Nakabayashi, K., \& Tsuchida, Y. (1995). Basic study on classification of fine particles in almost rigidly rotating flow (2nd report, dependence of Ekman layer and interior region on wall configuration of housing and rotor). Transactions of the Japanese Society of Mechanical Engineers, Part B, 61, 591, 3996.

Nakabayashi, K., Tsuchida, Y., Takeo, K., \& Ono, Y. (1995). Basic study on classification of fine particles in almost rigidly rotating flow (3rd report, conditions of occurrence of a stable regime of almost rigidly rotating flow). Transactions of the Japanese Society of Mechanical Engineers, Part B, 61, 591, 4004.

Pedlosky, J. (1969). Axially symmetric motion of a stratified, rotating fluid in a spherical annulus of a narrow gap. Journal of Fluid Mechanics, 36, 401.

Proudman, I. (1956). The almost-rigid rotation of viscous fluid between concentric spheres. Journal of Fluid Mechanics, 1, 505.

Schultz, D. H., Schwengels, S., Pralhad, R. N., \& Kocamustafaogullari, G. (1991). High-order method for heat transfer in a fluid between concentric rotating spheres. Computers \& Fluids, 20, 43. 